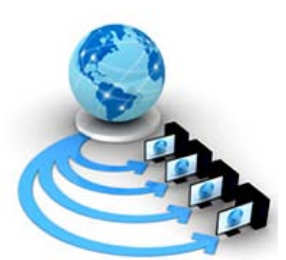

\title{
COLLABORATIVE CLUSTERING PROTOCOL IN WIRELESS SENSOR NETWORKS
}

\author{
Tewodrose Tilahun Dejen \\ Department of Computer Science and Systems Engineering \\ College of Engineering (A), Andhra University \\ Visakhapatnam-530 003, India
}

\author{
Professor Kuda Nageswara Rao \\ Department of Computer Science and Systems Engineering \\ College of Engineering (A), Andhra University \\ Visakhapatnam-530 003, India
}

\begin{abstract}
Cluster formation is one of the best strategies widely used for energy-constrained sensor nodes. The critical problem in clustering is the limit of a number of clusters formed and election of appropriate cluster heads. In this paper, we propose a novel collaborative clustering protocol in which two types of nodes will be nominated in each data gathering period, the central cluster head and highest energy node in the cluster. The central cluster head is nominated upon its proximity to cluster centroid and the highest energy node in the cluster sends the data towards the base station. The protocol is energy efficient in the sense that the most central node collects data from its member to reduce intracluster communication cost and highest energy node will face long-distance transmission towards the base station. Nomination of central cluster head is based on rotation schemes to distribute burdens in data collection and each nominated node will elect the next candidate central cluster head to enhance deterministic nature of head election mechanism. The proposed protocol is simulated using OMNeT++ and the result verifies $89 \%$ improvement in network lifetime compared to LEACH protocol.
\end{abstract}

Keywords: Clustering protocol; Collaborative cluster formation; Wireless sensor networks

\section{INTRODUCTION}

Wireless sensor network (WSN) is usually composed of a large collection of small autonomous sensor devices that can sense physical and environmental conditions. It is the most emerging technology used in a variety of applications such as industrial areas, home network, land and underwater disaster management, habitat monitoring, weather forecasting, medicine, military etc. Each sensor network has at least one base station where these sensor nodes send their data to the base station. A sink or base station is a nonenergy constraint or a device that has sufficient storage, processing capacity and acts as an interface between users and the network. Users will retrieve the required information from the network by injecting queries and gathering results from the sink. In most of these applications, wireless sensors are deployed statically [3]. In the highly dynamic and energy constraint network, it is a challenging task to develop a routing protocol.

WSNs development was originally initiated by military applications, The advancements in wireless communications, low-power [1] electronics manufacturing, embedded microprocessors and interesting attributes of sensor nodes such as tiny size, low-power, low-cost and Multifunction have made WSNs available for wide range of potential applications.

The wireless sensor networks are characterized by, inadequate computational power, poor storage capacity and limited, irreplaceable battery power, short-range radio communication, massive and random node deployment, unreliable environment, mobility nature of nodes and inexpensive compared to the traditional sensor.

Since sensor nodes are battery powered and have inadequate energy capacity, energy constraint is the biggest challenge/problem for network designers in harsh and hostile environments such as in a battlefield, where it is difficult to access the sensor nodes and recharge or replace their batteries [12]. There is a different type of applications that make that WSNs differ greatly from conventional networks like data or telecommunication Networks, network (MANET) and cellular systems. These networks have to operate in a self-organizing ad-hoc fashion since none of the nodes are likely to be capable of delivering the resources to act as a base station or central manager. All the nodes may not have its own a timeslot, when it is in one of the sleep modes, since being in a sleep mode is inherent to not transmitting traffic control section every frame.

Whenever the network is in communication, the nodes will be going to finish its energy or power. Therefore nodes will not function properly in which that will have a negative impact on the network performance. Therefore, routing protocols that need to be designed for sensor networks should be energy efficient so as to prolong its network lifetime. Cluster heads are responsible to forward incoming packets from other clusters there is a significant difference in energy dissipation among the cluster head nodes [7]. Which creates unbalanced energy consumption among the cluster head nodes.Cluster head selection plays a significant role in determining the lifespan of the sensor network.

Here are some of the methods that need to be done in selecting the appropriate cluster heads. The process of grouping the sensor nodes in a densely deployed large-scale sensor network is known as clustering. It involves grouping of nodes into clusters and electing a cluster head for wireless sensor networks [16].There are some issues involved with the process of clustering in a wireless sensor network. , how many clusters should be formed, how many of the nodes should be taken into a single cluster, the last important issue is the selection that used for the procedure of cluster-head in a cluster formations.

In any hierarchical routing protocol, selection of $\mathrm{CHs}$ is a very important step. Clustering can be done in two types of networks, homogeneous and heterogeneous networks on the basis of energy. [15, 24] Homogeneous are those in which nodes have same initial energy while heterogeneous networks are those in which nodes have different initial energy. Static clustering technique along with the heterogeneous distribution of nodes has been using prolong the stability period and network lifetime. In A heterogeneous sensor nodes, some of the nodes have more power than others. 
In Hierarchical clustering, the sensor nodes are organized into a hierarchy, based on their power levels and proximity. $[16,24]$. In these routing scheme nodes which has higher energy is supposed to be selected randomly selected for processing and sending data while low energy nodes are used for sensing and send information to the cluster heads. Clustering technique enables the sensor network to work more efficiently. It increases the energy consumption of the sensor network and hence the lifetime. Traditional routing protocols for WSN may not be optimal in terms of energy consumption.

Clustering techniques can be efficient in terms of energy and scalability. The objective of clustering is to minimize the total transmission power aggregated over the nodes. Every cluster selects a cluster head $(\mathrm{CH})$ responsible for coordinating the data transmission among the nodes in a cluster. Hierarchical Protocols are used to conserve energy by grouping the nodes into clusters. These are some hierarchical protocols, as LEACH [1], it is a protocol used for selection of cluster heads. But it doesn't take into account the distribution of sensor nodes and battery power. Multi-Hop LEACH is, modified [2] from $\mathrm{LEACH}$ uses cluster formation, elects $\mathrm{CH}$ and vice $\mathrm{CH}$ and Election of a vice $\mathrm{CH}$ is performed which performs the cluster formation and elects a $\mathrm{CH}$ and a vice $\mathrm{CH}$ which is used when the $\mathrm{CH}$ dies So even if the $\mathrm{CH}$ dies due to the gathering of data from all the nodes, cluster won't be useless as a Vice $\mathrm{CH}$ is there which will take the role of $\mathrm{CH}$ to transmit to the BS.

In this paper, we propose a novel Collaborative Clustering Protocol (CCP) that preserves the collaboration of central cluster head and highest energy node for energy efficiency. The basic philosophy behind the proposed protocol is that node which is very close to most of the nearby nodes on the average should collect local cluster data and node having highest energy in each cluster should assist central cluster head through transmission of data to long distance standing base station. The pillars behind the protocol are minimizing the overall energy cost in cluster data collection phase through letting the central node to most of the nodes to act like cluster head and sharing burdens of this central cluster head through the participation of data reporting towards the base station.

The protocol is energy aware in the sense that it lets only the highest energy node to participate in burden sharing. The protocol also ensures little variations on a number of cluster heads in each round as currently acting central cluster heads nominate next round central cluster head nodes.

\section{Routing schemes in wires sensor networks}

The WSN topology is highly dynamic caused by frequent node mobility. [9] As the network size grows highly in demand, data generated by one or more sources usually have to be routed through several intermediate nodes to reach the destination due to the limited range of each node's wireless transmissions. There will be a problem when intermediate nodes fail to forward the incoming message. To prevent this usually acknowledgments and retransmissions are implemented to recover the lost data. However, this generates a large amount of additional traffic and delays in the network. [23] Without using these schemes, the reliability of the system can also be increased by using multipath routing. Multipath routing allows the establishment of more than one path between source and destination and provides an easy mechanism to increase the likelihood of reliable data delivery by sending multiple copies of data along different paths without acknowledgment schemes.

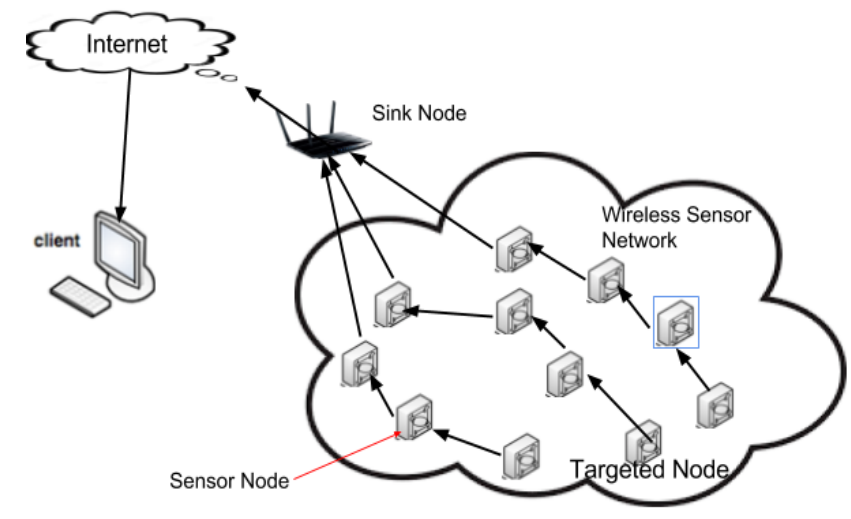

Figure1. Typical examples of multi hope wireless sensor networks

\section{Strategies of Cluster Head Selection}

As cluster head selection plays, the most important role of energy optimization in wireless sensor networks, we need to take care of appropriate cluster head selection for certain routing algorithms in wires sensor networks. We take a wider and brief comprising and summery the various cluster head section methods of hierarchical routing protocols that have the ability to maintain efficient energy utilization and data aggregation towards the base station [5, 15, 19, and 26]. Cluster formation is one of the best strategies widely used for energyconstrained sensor nodes, we need to select an appropriate number of clusters as well as cluster heads.

The location where the selected cluster heads are positioned plays a significant role in a network lifetime and affects the total energy consumption of the entire wireless sensor networks. Cluster heads can be dispersed in the sensor field randomly, or they can be deployed in a deterministic fashion. The following are some of the well-known method cluster head selection methods.

\subsection{Deterministic Schemes}

Sensor nodes select itself as a cluster head criterion during the communitarian process. In each round in order to make the decision of cluster head, hello packets are broadcast to all the sensor nodes of their neighbors. The first nodes to receive the pre-defined number of these messages declare themselves to be cluster head and the member nodes (ordinary nodes) will send "join me" request to the respective cluster heads. These techniques are very important and effective for energy optimization.

\subsection{Base Station Assisted Schemes}

The base station is a relay a short-range transceiver which connects cordless nodes, computer terminals, or another wireless device to a central hub and allows connection to a network. The base station by self-selects the appropriate cluster heads.

\subsection{Fixed-Parameter Probabilistic Schemes}

Cluster heads are chosen first due to the following 2 criteria's: By evaluating expressions involving:

- Using the Probabilistic methods requirements

- By using parameters like how many numbers of cluster heads are required.

\subsection{Resource Adaptive Probabilistic Schemes}

The threshold is calculated by the scheme taking into consideration the residual energy, energy consumed during the present round and the average energy of the node as additional 
parameters. This causes the strategy for the selection of the cluster head to be energy adaptive.

\subsection{Cluster Head Selection in Hybrid Clustering (Combined Metric) Schemes}

This scheme adjusts the nodes and the threshold function, and the non cluster-heads select the optimal cluster-head by taking into consideration the comprehensive nodes' residual energy and how far the node is from the base station.

\section{LITERATURE REVIEW}

A number of clustering protocols have been explored in order to obtain the effective energy usage in wireless sensor network. [22] The main goal of a sensor network is to forward the sensing data gathered by sensor nodes to the base station. One of the common techniques is direct data transmission. In this case, each node in the network directly sends sensing data to the base station. However, if the base station is remote from the sensor node, the node will soon die due to excessive energy consumption for delivering data. In order to solve this problem, some of the clustering algorithms aimed at saving energy have been proposed like leach (low-energy adaptive clustering $[1,4,14,21]$. hierarchically it is based on the randomized rotation of the cluster head to distribute the energy load among the sensor nodes evenly distribute in the entire network.

LEACH serves as the benchmark for most of the hierarchical clustering algorithm for WSNs that was developed for reducing power consumption. [1] It is self-adaptive and self-organized. LEACH protocol uses round as unit, each round is made up of cluster set-up stage and steady-state stage, for the purpose of reducing unnecessary energy costs, the steady-state stage must be much longer than the set-up stage each node elects itself as a cluster head based on a probabilistic scheme and broadcasts its availability to all the sensor nodes present in the area. During the phase of clustering, the cluster head plays an important role in providing data communication to nodes and delivered the base station efficiently. In this protocol each node has equal probability becoming a cluster head.

In Leach, the clustering task is distributed among the nodes based on duration. Direct communication is used by each cluster head (ch) to forward the data to the base station (BS).

Nodes will generate a random number between 0 and 1 and they will be compared with the threshold value. The node becomes a cluster head for the current round if the number is less than the threshold value (TN).

HEED (hybrid energy efficient distributed efficient distributed clustering) is primarily designed to achieve power balancing by extending the leach protocol. [2]. In HEED algorithm, the residual energy is playing an important role in determining the possibility of nodes becoming $\mathrm{CH}$.

HEED improves the basic scheme of LEACH based on residual energy as parameters for cluster selection to accomplish power balancing. The protocol is serving in multi-hop networks, using an adaptive transmission power for inter-cluster communication. In HEED, the selection of a $\mathrm{CH}$ is based on two parameters, viz, Residual Energy and the proximity of the neighbor nodes.

This algorithm proposes a new approach of an energyefficient homogeneous clustering algorithm for wireless sensor networks uses selection criteria's like holdback value, the residual energy of existing cluster heads, and nearest hop distance of the in which the lifespan of the network is increased by assuring a homogeneous distribution of nodes in the clusters. [20] Therefore algorithm, will improve the energy efficiency and network performance through selecting cluster heads on the node
It also introduces a new clustering parameter for cluster head election, which can better handle the heterogeneous energy capacities. Power efficient routing in wireless sensor network is the main challenge for researchers. This protocol deals with cluster formation based on different techniques and criteria such as cluster head selection, aggregation of the sensed data within a cluster and sending that to the base station in an energy efficient way.

The smallest distance between cluster heads in a cluster based sensor network is prolonging network lifetime by dispersing the cluster heads, thus lowering the average communication energy consumption [4].

This protocol is used for sensing the temperature applications in a hierarchical approach including with the use of data-centric schemes. The cluster head broadcasts two thresholds to the nodes [14]. TEEN is not recommended for applications where periodic reports are needed since the user may not get any data at all if the thresholds are not reached. [6] It is observed that with increasing number of levels of nodes energy saving also increases Load balancing using clustering method is one of the most practical solutions, regarding to energy limitation in wireless sensor networks.

This study, propose that a new energy efficient (EE) clustering based protocol for single-hop, heterogeneous WSN [24].The newly proposed protocol uses channel state information (CSI) in the selection process of Cluster Heads so as to improve and showing better stability period than that of well-known protocols than existing routing protocols.

Separate clusters are created using the cluster creation algorithm. [9] Based upon the quality of services as metrics available information with each sink node, data packets are routed to the base station. Due to the hierarchical architecture, the performance is unaffected by the increase in the number of mobile nodes, at the same time the Packet Loss is reduced. This paper proposes a new clustering approach MZ-SEP based on multiple triangle zones distribution and SEP protocol. The partition of sensor deployment field into multiple zones enhances the communication between cluster heads and their members [17]. Hierarchical Clustering is an energy-efficient communication protocol which can be used by the sensors to report their sensed data to the sink. [11] The routing protocol of wireless sensor network must minimize the energy consumption so as to increase the network lifetime. Many researchers have already proposed a number of distinct algorithms based on different technique the Hierarchical clustering algorithms are the most important ones.

This article proposes Unequal Clustering Size (UCS) designed for more uniform energy dissipation among the cluster head nodes. [13]. In one-hop communication, every sensor node can directly reach the destination, while in multihop communication, nodes have transmission range and therefore are forced to route their data over several hops until the data reach the final destination. Manjeshwar proposed Threshold sensitive Energy Efficient sensor Network protocol (TEEN)

Hansen, Ewa proposes that the geographical distribution of the cluster heads severely influences the overall energy consumption of the network [5]. Spreading the cluster heads more evenly means prolonging the lifetime of the network. In this article, a distributed randomized clustering algorithm for generating a hierarchy of CHs is proposed

In this paper, the authors propose a novel clustering algorithm, Front-Leading Energy Efficient Cluster Heads (FLEECH), in which the whole network is partitioned into regions with diminishing sizes [15]. In each region, we form multiple clusters. Here in this proposed routing algorithm cluster head selection is based on residual energy and distance 
of each node to the sink as the most significant parameter [16]. This article is based on the concept of finding the cluster head minimizing the sum of Euclidean distances between the head and member nodes.

This protocol operates based on local information .here there is no demand for centralized control and produces relatively small communication overhead [10]. This paper introduces energy efficient and density control clustering algorithm (EEDCA) [18]. On this proposed approach, the selection of the cluster head depends on residual energy, density, and distance.

The author proposed an efficient algorithm, which is mainly considering the energy density of the clusters to balance the energy and prolong the network lifetime [19, 25]. A unique two-step cluster head method is devised to select the proper cluster head and the shortest path selection from source to sink is performed by using On-demand routing approach.

B. Elbhiri assumptions are based on dividing the network into dynamic clusters [30].The cluster's nodes communicate with an elected node called cluster head, and then this cluster head aggregates and communicates the information to the base station

The proposed algorithm guarantees the entire network stays alive for a longer time than the other existing energy efficient techniques based on residual energy for the selection of cluster heads. This paper proposes energy-aware routing protocol (EAP) for a long-lived sensor network and the protocol improves the lifetime of the entire network by minimizing energy consumption for in-network communications and balancing the energy load among sensor nodes [3].

This study further explains more about routing methods of Enhanced Developed Distributed Energy-Efficient Clustering scheme (EDDEEC) for heterogeneous Wireless sensor networks [21]. The algorithm uses the techniques of changing dynamically Cluster Head $(\mathrm{CH})$ election probability.

Arati Manjeshwar proposes a hybrid routing protocol (APTEEN) which allows for comprehensive information retrieval. [29] The nodes in such a network not only react to time-critical situations but also give an overall picture of the network at periodic intervals in a very energy efficient manner.

This work is a comprehensive and fine-grained survey on clustering routing protocols from the proposed literature for wires sensor networks [22]. This outline has the advantages and objectives of clustering for WSNs and develops a novel taxonomy of WSN clustering routing methods based on complete and detailed clustering attributes.

This paper presents a clustering scheme to create a hierarchical control structure for multi-hop wireless networks. Hierarchical routing algorithms are the best mechanisms that have been used to provide for highly scalable opportunities in many large networking systems that have been designed [23]. It primarily demonstrates how certain geometric properties of the wireless networks can be exploited to perform clustering with some desired properties.

Power-Efficient Gathering in Sensor Information Systems (PEGASIS) is a near optimal chain-based protocol [8]. In order to extend network lifetime, nodes need only communicate with their closest neighbors and they take turns in communicating with the base station and when the round of all nodes communicating with the base-station ends, the new round will start.

\section{PROPOSED STRATEGIES AND ALGORITHMS}

The major aim of this paperwork is to maximize the network lifetime of sensor networks by lowering the total energy dissipation needed for delivering data to the base station. Hierarchical-based routing is a cluster based routing in which high energy nodes are randomly selected for processing and sending data while low energy nodes are used for sensing and send information to the cluster head. The main objective of clustering is to minimize the total transmission power aggregated over the nodes. Every cluster selects a cluster head (CH) responsible for coordinating the data transmission among the nodes in a cluster to deliver to the base stations.

Noncluster head nodes do not transfer data directly to the base station node rather they send the sensed data to the cluster head. CHs aggregate data it received from its member nodes and forward it to the base station. Therefore the number of nodes communicated to the base station will be decreased and the total energy dissipation significantly reduced.

Basic assumptions taken in the proposed routing protocol are the following:

- $\quad$ Sensor nodes are homogenous having the same initial energy, processor power, and memory capacity.

- The transmission power level of sensor nodes can reach the base station which is located outside of network area.

- Nodes have built-in GPS for location information

- Ideal MAC layer and error-free communication channel.

Initially, each sensor nodes will send location information to the base station and then the base station will nominate nodes to act as central cluster heads (CCH nodes). Election of $\mathrm{CCH}$ nodes is upon computation of the density of neighbor nodes within transmission region and nodes having a high density within transmission range are center to most nodes, hence elected as $\mathrm{CCH}$ nodes. Base station elects $\mathrm{CCH}$ nodes only once and any other election of $\mathrm{CCH}$ nodes in subsequent rounds will be decided locally without the participation of base station.

Then CCH nodes will send HEAD_ADVERT message to all neighbor nodes within competition radius. Upon reception of HEAD_ADVERT messages from CCH nodes, regular nodes decide their own $\mathrm{CCH}$ node based on distance cost metric and they will send a HEAD_JOIN message to respective $\mathrm{CCH}$ nodes embedding their within residual energy and location information the network area. The $\mathrm{CCH}$ nodes receiving HEAD _JOIN message compute the cluster centroid based on location information of nodes and the highest energy node (HEN) within the cluster. Cluster member very close to cluster centroid will be nominated as the candidate $\mathrm{CCH}$ node for next round. $\mathrm{CCH}$ nodes pass the decision on the election of HEN nodes upon the prediction of energy remaining after each cluster member send their own packet to $\mathrm{CCH}$ node for the entire TDMA frames. So in each round of data gathering period, CCH nodes collect data from cluster members, aggregate the correlated data and send it to HEN nodes .then the HEN node sends the received data directly to the base station. The collaboration between $\mathrm{CCH}$ and HEN nodes is quite logical in the sense that the most central node should collect local data from cluster members for minimizing intracluster communication cost and node having the highest energy should collaborate with $\mathrm{CCH}$ node to face long-distance transmission to the base station and share burdens imposed on cluster head nodes (CCH nodes) in effect. Let $(x i, y i)$ be the position of the sensor node, the cluster centroid $(x c, y c)$ can \[ y c=\frac{\sum_{i=1}^{N} y i}{N c h i l d} \text { Where the symbol } \]
be calculated a, and
Nchild defines the number of cluster member of CCH node. 
Computation of the cluster centroid based on the position of member nodes and the nominating node with minimum distance from this centroid as $\mathrm{CCH}$ node for next round increase the possibility of $\mathrm{CCH}$ nodes to be located in a region which is very close to each and every nearby node and then reducing the overall energy cost in intracluster communication. The protocol is to ensure that elected cluster head node should reduce local data collection cost and HEN node within the cluster is expected to share burdens of $\mathrm{CCH}$ node through the participation of long-distance transmission towards the base station.

The proposed protocol also eliminate random nature of cluster head election mechanism as current $\mathrm{CCH}$ nodes nominate $\mathrm{CCH}$ nodes for next round based on distance from cluster centroid. Hence the number of cluster heads elected in each round is deterministic compared to an unpredictable number of cluster heads in LEACH protocol. Election of $\mathrm{CCH}$ nodes is based on rotation to distribute energy consumption in cluster data collection and because of this sometimes nodes with a few number of neighbor nodes may be elected as $\mathrm{CCH}$ node. In such scenario, the current $\mathrm{CCH}$ node will not be involved in next $\mathrm{CCH}$ election process as cluster centroid computed from a few number of nodes and nomination of next round $\mathrm{CCH}$ accordingly reduce the fairness of burdens among $\mathrm{CCH}$ nodes. Regular nodes which are not receiving any HEAD_ADVERT message nominate themselves as $\mathrm{CCH}$ node and inform nodes within transmission range. Node nominating itself as $\mathrm{CCH}$ node but does not receive any HEAD_JOIN message from nearby nodes will quit from its wish of being $\mathrm{CCH}$ and it will join one of $\mathrm{CCH}$ nodes upon reception of advertisement message from newly nominated $\mathrm{CCH}$ nodes.

\section{Algorithm (cluster formation)}

\begin{tabular}{|l|}
\hline Nodes send location information to BS \\
The bs nominates and announce NOPT CCH nodes \\
for each node receiving bs announcement \\
if(nominated as CCH node) \\
broadcast HEAD_ADVERT msg \\
endif else \\
wait for CCH node adverts \\
end for for each node receiving HEAD_ADVERT msg \\
identify its CCH node \\
send HEAD_JOIN to CCH node (residual \\
energy \& location) \\
end for \\
for each node receiving HEAD_JOIN msg \\
if(currently CCH node) \\
compute cluster centroid \\
nominate CCH node for next round \\
identify HEN node \\
endif \\
end for
\end{tabular}

\section{Energy dissipation model}

Energy dissipation is the most important deciding factor to determine the life of a sensor network because usually sensor nodes are driven by a battery. Accurate estimation of sensor network lifetime requires the precise energy consumption model.

Energy dissipation by sensor nodes for data transmission has a direct relation to the transmission distances and data packet size to be transmitted. Sometimes energy optimization is more complicated in sensor networks because it involved not only reduction of energy consumption but also prolonging the life of the network as much as possible. Heizelman [1] proposed a model that considers microcontroller Processing and radio transmission and receiving only. This model does not consider other important sources of energy consumption, such as transient energy, sensor sensing, sensor logging and actuation accurate.

The energy dissipates by a node in transmitting N-bit packet to another node which is located at distance of $S$, is given by:

$\mathrm{E}_{\mathrm{TX}}(\mathrm{N}, \mathrm{S})=\left\{\begin{array}{cc}\mathrm{N} * \mathrm{E}_{\text {elec }}+\mathrm{N} * \mathrm{E}_{\mathrm{fs}} * \mathrm{~S}^{2} & \text { if } \mathrm{S}<d 0 \\ \mathrm{~N} * \mathrm{E}_{\text {elec }}+\mathrm{N} * \mathrm{E}_{\mathrm{amp}} * \mathrm{~S}^{4} & \text { if } \mathrm{S} \geq \mathrm{d} 0\end{array}\right.$

For reception of $\mathrm{N}$ packets, the radio dissipation model expenditure can be calculated as:

$$
E_{R X}(\mathrm{~N})=E_{\text {elec }} * \mathrm{~N}
$$

Where N, S, do represent a number of the packets of bits, transmission distances, and the threshold distances respectively

$$
[27,28] \text {. }
$$

The electronics energy Elec depends on factors such as:

- Filtering

- Digital coding

- Modulation and

- Spreading of the signal

Whereas the amplifier energy, Efs.d2 or Eamp.d4, depends on the distance to the receiver and the acceptable bit-error rate Value of threshold distance do can be calculated as:

$$
d_{0}=\frac{E_{f s}}{E_{m p}}
$$

If the distance is less than a threshold do, the free space model is used otherwise, the multipath model is used. In our scenario, we use both of the two-channel models based upon the distance between the transmitter and receiver [1, 26]. For the verification of the energy efficiency of the proposed protocol, we need to take some important modeling features in the next subtopics.

\section{Network Model}

Here is a list of assumptions are considered about the model of the network for the proposed protocol that we are going to develop.

a) The nodes are randomly\& uniformly distributed in the reign.

b) Noncluster head nodes do not transfer data directly to the base station.

c) The Base Station (BS) which is placed and located at a fixed distance far away from network filed where nodes are deployed. It is a resource-rich device that has adequate storage and processing power

d) Sensor nodes are left unattended once deployed randomly in the area of interest.

e) Nodes are battery operated and homogeneous with inform initial energy allocation. The battery could not replace or not be rechargeable.

f) All sensors have location determination devices such as GPS.

g) Nodes that are found close to one another may have similar or correlated data. Sensor nodes should be time synchronized in the order of a second.

h) The sink node possesses the highest energy and is static. 


\section{IV.SIMULATION RESULTS AND ANALYSIS}

The performance of the proposed protocol is evaluated using OMNeT++network simulator and the result of the simulations stored in vector and scalar files are analyzed using MATLAB software. We have assumed that the underlying MAC layer is ideal and the communication channel is free of error. We define network lifetime based on around at which first sensor node dies (FND) as this metric measures the stability period of sensor networks. The proposed protocol is compared with low energy adaptive clustering hierarchy (LEACH) protocol based on a "round" at which the first node dies (FND), 20\% of node dies (PND), half node dies (HND) and the last node dies (LND). The number of sensor nodes alive per each round and variance of energy consumption among sensor nodes area also examined in the simulations. The simulation parameters are summarized in table 1. As shown in Fig.1, the nodes are randomly\& uniformly distributed and the base station is located outside of the region under investigation in the simulation. Table 2 shows FND, PND, HND and LND performance metrics in nutshell. The proposed protocol has \% improvement in network lifetime which shows that the stability period of CPP protocol is elongated compared to LEACH protocol. 20\% of nodes die at round 1007 \& 1303 in LEACH and CPP protocol respectively. The duration between FND \& LND (instability period) in CPP protocol is short in length compared to LEACH protocol implying that energy consumption of sensor nodes is relatively homogenous in our protocol which avoids the early death of sensor nodes.

Table 1.Simulation Parameters

\begin{tabular}{|c|c|c|c|}
\hline Parameter & Value & $\begin{array}{l}\text { Parame } \\
\text { ter }\end{array}$ & Value \\
\hline $\begin{array}{l}\text { Network } \\
\text { area }\end{array}$ & $\begin{array}{ll}(0,0) & \text { to } \\
(100,100) & \\
\end{array}$ & efs & 10pJ/bit/m2 \\
\hline $\begin{array}{l}\text { Number of } \\
\text { nodes }\end{array}$ & 45 & emp & $\begin{array}{l}0.0013 \mathrm{pJ} / \mathrm{bit} / \\
\mathrm{m} 2\end{array}$ \\
\hline $\begin{array}{l}\text { Initial } \\
\text { energy }\end{array}$ & $2 \mathrm{~J}$ & eda & $5 \mathrm{~nJ} / \mathrm{bit}$ \\
\hline BS location & $(125,50)$ & $\begin{array}{l}\text { Cont } \\
\text { rol } \\
\text { packet } \\
\text { size }\end{array}$ & 200 bits \\
\hline Elec & 50nJ/bit & $\begin{array}{c}\text { Data } \\
\text { packet } \\
\text { size }\end{array}$ & 4000 bits \\
\hline do & $87 \mathrm{~m}$ & $\begin{array}{c}\text { TD } \\
\text { MA } \\
\text { frames }\end{array}$ & 3 \\
\hline
\end{tabular}

Table 2.FND, PND, HND, AND LND

\begin{tabular}{|l|r|l|l|l|}
\hline Protocols & \multicolumn{1}{|l|}{ FND } & PND & HND & LND \\
\hline LEACH & 477 & 1007 & 1496 & 2397 \\
\hline CPP & 903 & 1303 & 1432 & 1682 \\
\hline
\end{tabular}

The number of sensor nodes working till all nodes dies in target region affects the degree of data gathering and the functionality of wireless sensor networks. Fig.2 depicts the number of alive nodes per each round. The simulation manifests that the CPP protocol has superior performance compared to LEACH protocol till $62 \%$ of sensor nodes are alive.Fig.4\&5 show the number of elected cluster heads for each round in LEACH and CCP protocol respectively. There is high variation in a number of cluster heads in each round for LEACH protocol because of random nature of cluster head election whereas the variation in CCP protocol is very slow as currently acting cluster heads nominate the next candidate cluster heads in each data gathering period. The simulation shows that nomination of cluster heads in CCP protocol is relatively deterministic compared to $\mathrm{LEACH}$ protocol which is used to maintain an optimum number of cluster heads for all data gathering periods.

In CPP protocol, the $\mathrm{CCH}$ nodes are centered to most of the cluster members and hence the average energy consumption of nodes in intracluster communication seems relatively homogenous in theory compared to LEACH protocol which blindly selects cluster heads probabilistically without considering the centrality of the nominated cluster head. In addition, the highest energy node in cluster share burdens imposed on CCH nodes for the bulky energy loss accompanied after long-distance transmission to the base station and theoretically this allows nodes to have a good distribution of energy loss for each data gathering period in effect. To see the status of homogeneity in energy consumption among sensor nodes, we compute the standard deviation of residual energy of nodes per each round till all sensor nodes are flying to death. The simulation result in Fig.6 justifies the theoretical explanation and our protocol has relatively uniform energy consumption among nodes compared to LEACH protocol.

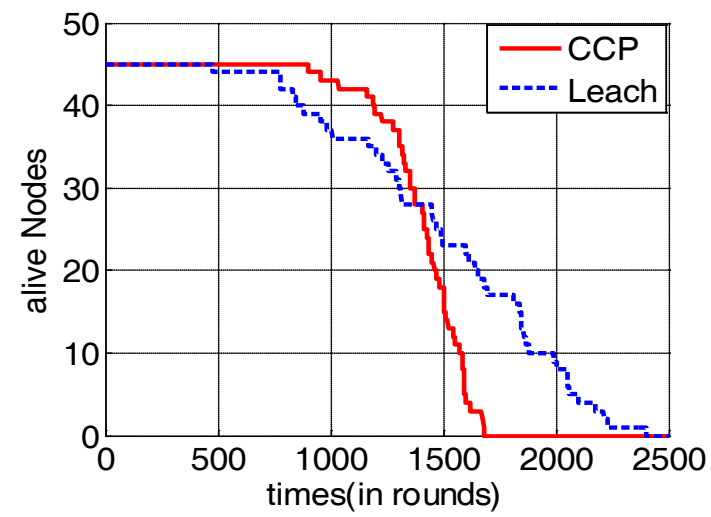

Fig.3 Alive nodes per rounds

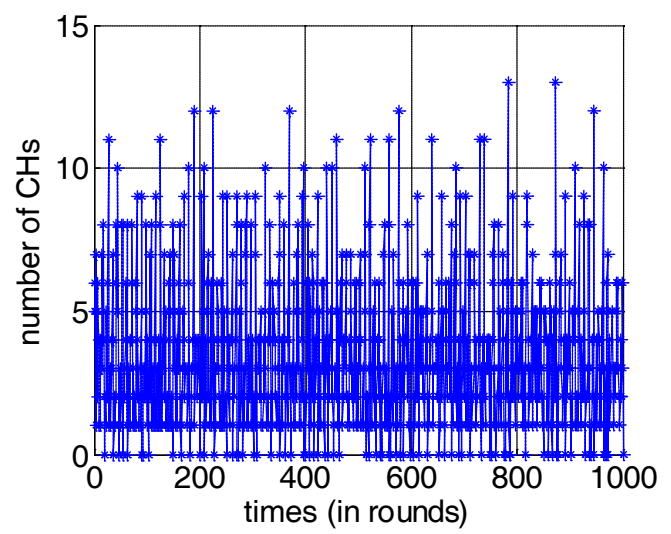

Fig.4: Number of CHs per round in LEACH protocol 


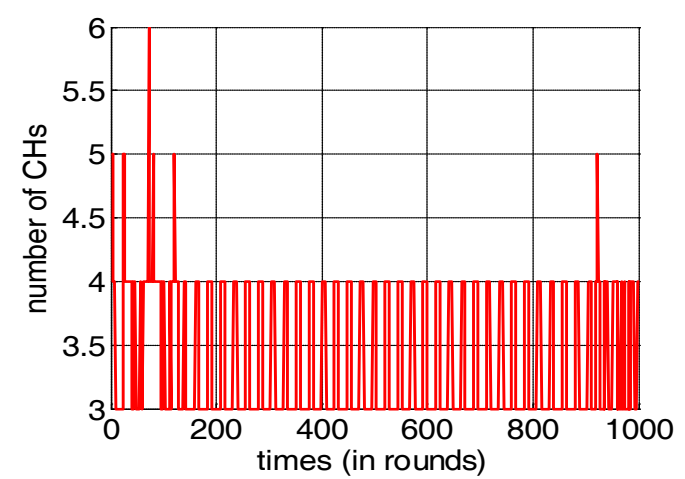

Fig.5: Number of CCHs per round in CCP protocol

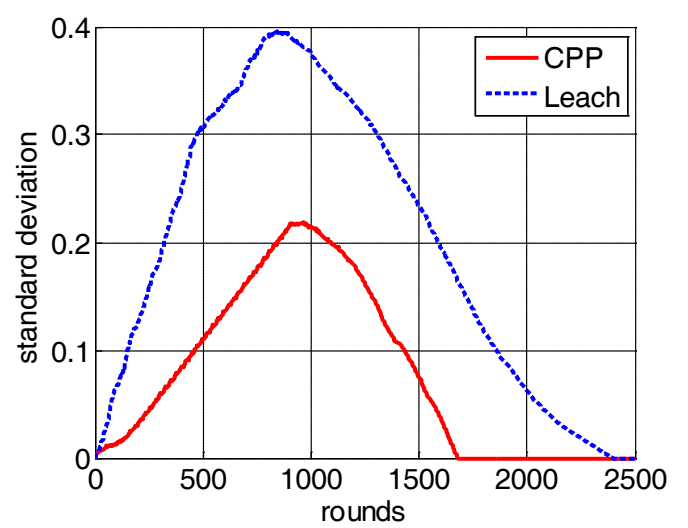

Fig.6 Residual energy standard deviation among node

\section{V.CONCLUSION}

In order to extend the lifetime of wireless sensor networks, many approaches have been done. Wireless Sensor Networks dividing the network into clusters, gathering data from nodes and aggregating them to the base station. Some of the clustering algorithms consider the residual energy of the nodes in the selection of the cluster heads and others rotate the selection of cluster heads periodically.

The network lifetime of energy sensitive wireless sensor networks relies on efficient clustering algorithm. In this paper, we propose energy efficient clustering protocol in which the central cluster head and the highest energy node in the cluster collaboratively send the data towards the base station. The proposed protocol also avoids random cluster head election mechanism and simulation results manifest that it has better performance compared to LEACH protocol.

\section{V1.REFERENCES}

W.Heinzelman,

A.ChandrakasanandH.Balakrishnan,"Energy-efficient communication protocols for wireless microsensor networks" Proceedings of the HawaiiInternational Conference on Systems Sciences, Jan 2000.

[2] Younis, Ossama, and Sonia Fahmy. "HEED: a hybrid, energy-efficient, distributed clustering approach for ad hoc sensor networks." IEEE Transactions on mobile computing 3, no. 4 (2004): 366-379.

[3] Liu, Ming, Jiannong Cao, Guihai Chen, and Xiaomin Wang. "An energy-aware routing protocol in wireless sensor networks." Sensors 9, no. 1 (2009): 445-462

[4] Roy, Sohini, and Ayan Kumar Das. "Energy-efficient cluster-based routing protocol (EECBRP) for Wireless Sensor Network." In Networks \& Soft Computing
(ICNSC), 2014 First International Conference on, pp. 2529. IEEE, 2014.

[5] Hansen, Ewa, Jonas Neander, Mikael Nolin, and Mats Björkman. "Energy-efficient cluster formation for large sensor networks using a minimum separation distance." In The Fifth Annual Mediterranean Ad Hoc Networking Workshop, vol. 6. 2006.

[6] Sabet, Maryam, and Hamid Reza Naji. "A decentralized energy efficient hierarchical cluster-based routing algorithm for wireless sensor networks." AEU-International Journal of Electronics and Communications 69, no. 5 (2015): 790799.

[7] Muhammad Arshad, Mohamad Y.Aalsalem, Farhan A. SIDDIQUI "energy efficient cluster head selection in mobile wireless sensor networks" Journal of Engineering Science and Technology Vol. 9, No. 6 (2014) 728 - 746

[8] Jamal N. Al-Karaki Ahmed E. Kamal "Routing Techniques in Wireless Sensor Networks” A Survey

[9] Narendra Reddy Mandhadi1 and Lakshmi Rajamani2 “QoS aware Hierarchical Multi-hop routing schemes for mobile ad hoc networks", International Journal of Computer Science \& Engineering Survey (IJCSES) Vol.4, No.1, February 2013.

[10] Ataul Bari, Arunita Jaekel and Subir Bandyopadhyay "Distributed Clusterin Around Relay Nodes in Sensor Networks"

[11] Puneet Gurbani, Hansa Acharya, Prof. Anurag Jain,"Hierarchical Cluster-Based Energy Efficient Routing Protocol for Wireless Sensor Networks": An (IJCSIT) International Journal of Computer Science and Information Technologies, Vol. 7 (2), 2016, 682-687

[12] Stefan Dulman, Lodewijk v. Hoesel, Tim Nieberg, Paul Havinga,"Collaborative communication protocols for wireless sensor networks"

[13] Soro, Stanislava, and Wendi B. Heinzelman. "Prolonging the lifetime of wireless sensor networks via unequal clustering." In Parallel and Distributed Processing Symposium, 2005. Proceedings. 19th IEEE International, pp. 8-pp. IEEE, 2005.

[14] Bhovi, Ajit K., and Gopal A. Bidkar. "TEEN Protocol in Wireless Sensor Network." Bonfring International Journal of Research in Communication Engineering 6, no. Special Issue Special Issue on Recent Advancements in Electronics and Communication Engineering| Editors: Dr. GA Bidkar, Dr. C. Vijaya and Dr. SB Kulkarni (2016): 01-03. (PDGC), 2010 1st International Conference on, pp. 205-210. IEEE, 2010.

[15] Nayak, Basanta K., Monalisa Mishra, Satyananda Champati Rai, and Sateesh K. Pradhan. "A novel cluster head selection method for energy efficient wireless sensor network." In Information Technology (ICIT), 2014 International Conference on, pp. 53-57. IEEE, 2014.

[16] Park, Geon Yong, Heeseong Kim, Hwi Woon Jeong, and Hee Yong Youn. "A novel cluster head selection method based on K-means algorithm for energy efficient wireless sensor network." In Advanced Information Networking and Applications Workshops (WAINA), 2013 27th International Conference on, pp. 910-915. IEEE, 2013.

[17] Mahboub, Aziz, Mounir Arioua, Imad Ez-Zazi, and Ahmed El Oualkadi. "Multi-zonal approach clustering based on stable election protocol in heterogeneous wireless sensor networks." In Information Science and Technology (CiSt), 2016 4th IEEE International Colloquium on, pp. 912-917. IEEE, 2016.

[18] Meddah, Meriem, Rim Haddad, and Tahar Ezzedine. "An Energy Efficient and Density control Clustering Algorithm for Wireless Sensor Network." In Wireless Communications and Mobile Computing Conference 
(IWCMC), 2017 13th International, pp. 357-364. IEEE, 2017.

[19] Dongare, Snehal P., and R. S. Mangrulkar. "An improved cluster head selection approach for energy efficiency in wireless sensor networks: A review." In Pervasive Computing (ICPC), 2015 International Conference on, pp. 1-6. IEEE, 2015

[20] Singh, Shio Kumar, M. P. Singh, and D. K. Singh. "Energyefficient homogeneous clustering algorithm for wireless sensor network." International Journal of Wireless \& Mobile Networks (IJWMN) 2, no. 3 (2010): 49-61.

[21] Javaid, Nadeem, T. N. Qureshi, A. H. Khan, Adeel Iqbal, E. Akhtar, and M. Ishfaq. "EDDEEC: Enhanced developed distributed energy-efficient clustering for heterogeneous wireless sensor networks." Procedia Computer Science19 (2013): 914-919.

[22] Liu, Xuxun, "A survey on clustering routing protocols in wireless sensor networks." sensors 12, no. 8 (2012): 1111311153.

[23] Banerjee, Suman, and Samir Khuller. "A clustering scheme for hierarchical control in multi-hop wireless networks." In INFOCOM 2001. Twentieth annual joint conference of the IEEE computer and communications societies. Proceedings. IEEE, vol. 2, pp. 1028-1037. IEEE, 2001.

[24] Nuray, A. T., and S. M. Daraghma. "A New Energy Efficient Clustering-based Protocol for Heterogeneous
Wireless Sensor Networks." Journal of Electrical \& Electronics 4, no. 3 (2015).

[25] BM Thippeswamy, Reshma S, Shaila K, Venugopal K R, S S Iyengar And L M Patnaik4”On-Demand Cluster Routing Based On Energy Density In Wireless Sensor Networks".

[26] Maraiya, Kiran, Kamal Kant, and Nitin Gupta. "Efficient cluster head selection scheme for data aggregation in wireless sensor network." International Journal of Computer Applications 23, no. 9 (2011): 10-18.

[27] Akkaya, Kemal, and Mohamed Younis. "A survey on routing protocols for wireless sensor networks." Ad hoc networks 3, no. 3 (2005): 325-349.

[28] Halgamuge, Malka N., Moshe Zukerman, Kotagiri Ramamohanarao, and Hai L. Vu. "An estimation of sensor energy consumption." Progress In Electromagnetics Research B 12 (2009): 259-295.

[29] Manjeshwar, Arati, and Dharma P. Agrawal. "APTEEN: A hybrid protocol for efficient routing and comprehensive information retrieval in wireless sensor networks." In ipdps, p. 0195b. IEEE, 2002

[30] Bathla, Gaurav. "Minimum Spanning Tree-based Protocol for Heterogeneous Wireless Sensor Networks." i-Manager's Journal on Wireless Communication Networks 1, no. 4 (2013): 12. 